\title{
Vector Field Matrix Derivation on a Recursive Algorithm of Six Degrees of Freedom Contour Matching
}

\author{
${ }^{\circ}$ Toshiyuki AOKI* and Kohji KAMEJIMA* \\ * Mechanical Engineering Research Laboratory, Hitachi, Ltd \\ 502 Kandatsu, Tsuchiura, Ibaraki 300, Japan
}

\begin{abstract}
This paper presents a vector field matrix [2] derivation on a recursive algorithm of six degrees of freedom contour matching through single-eye imagery. We have already reported an algorithm in which the position and orientation of an object is determined by matching the reference pattern to the edge pattern on the image. However, in that algorithm, the vector field matrix was given intuitively. Here we actually derived the vector field matrix.

First, a mathematical model of the potential field is formulated by the stochastic evolution equality in Hilbert space. The existence and uniqueness properties of the solution to the potential field equations are studied. Second, we consider the cost function and derive a pattern matching algorithm suitable for computers within the framework of the gradient method. Third, we derive the vector field matrix for mathematical security of the algorithm. Finally, a representative time result of the experiments is shown, to support the theoretical aspects developed here.

Key Words: Six Degrees of Freedom Contour Matching, Optical Flow, Potential Field, Vector Field Matrix
\end{abstract}

\section{Introduction}

Pattern detection has recently become important in surveying systems and robot operating systems. For example, surveying systems that use images need a matching algorithm to calculate the position and orientation of objects in construction sites and workshops. Robot operating systems need to know the environment around the robot. Therefore, a system measures the position and orientation of the object manipulated by the robot.

Many researchers have worked on pattern detection. We took particular note of pattern matching using the pattern field. Kamejima, Ogawa and Nakano reported pattern detection in random fields by considering the potential field [2]. In their work, two parameters of the position of an object were estimated. Hamada, Kamejima and Takeuchi considered power and moment in the potential fields and estimated three parameters of the position and orientation of the object [3]. We reported six degrees of freedom contour matching through single eye noisy imagery [5] and we estimated six parameters, all position and orientation of the object. However, for this algorithm, the derivative of the reference pattern with respect to the position and orientation of the object must be calculated, a process which was timeconsuming. The position and orientation of the object was given by an algorithm in which the potential field of the edge pattern and the reference pattern were used [6]. However, in the algorithm, the vector field matrix was given intuitively. In this paper, for the purpose of mathematical security of the algorithm, the vector field matrix is actually derived.

This paper is organized as follows: In Section 2, we describe the mathematical model for the potential field of the edge pattern and the reference pattern. The existence and uniqueness properties of the solution to the potential field equations are stated within the framework of function spaces. In Section 3, we consider the cost function and derive the six degrees of freedom matching algorithm, using the gradient method. In this algorithm, it is possible to obtain a convergent parameter at which the cost function has a local minimum. In Section 4, we guarantee the pattern matching algorithm by deriving the vector field matrix. In Section 5, a practical implementation algorithm of pattern matching is proposed by experiments, based on the definite difference method.

The principal symbols used here are listed below.

$T$ : time interval $] 0, \mathrm{t}_{\mathrm{f}}\left[\right.$, where $\mathrm{t}_{\mathrm{f}}$ is a constant

G: image domain $] 0, g_{1}[\times] 0, g_{2}\left[\right.$, where $g_{1}$ and $g_{2}$ are constants

$\Omega$ : sample space

$\mathrm{L}^{2}(\mathrm{G})$ : space of square integrable functions on $\mathrm{G}$

$\mathrm{H}^{\mathrm{m}}(\mathrm{G})$ : Sobolev space on $\mathrm{G}$ of order $\mathrm{m}$

$\mathrm{H}_{0}^{1}(\mathrm{G})$ : space $\left\{\phi \mid \phi(\mathrm{x})=0, \mathrm{x} \in \partial \mathrm{G}, \phi \in \mathrm{H}^{1}(\mathrm{G})\right\}$

$(\bullet, \bullet),\|\bullet\|$ : an inner product and a norm in $\mathrm{L}^{2}(\mathrm{G})$ respectively

$\mathrm{L}^{2}(\mathrm{X} ; \mathrm{Y})$ : space of function $\mathrm{f}$ such that

$$
\int_{G}\|f(x)\|_{Y} d x<\infty
$$

$\mathrm{C}(\mathrm{X} ; \mathrm{Y})$ : space of continuous functions from $\mathrm{X}$ to $\mathrm{Y}$ $\mathcal{L}^{1}(\mathrm{X} ; \mathrm{Y})$ : class of trace operators from $\mathrm{X}$ to $\mathrm{Y}$

\section{Mathematical Model}

First, we show an edge pattern. The edge pattern is obtained from the image by the zero-cross method [1]. This edge pattern $f_{m}$ is represented by the following function: 


$$
f_{m}=\{x \in \Omega:|\Delta f(x)| \leq \mu\},
$$

where $\Delta$ is the two-dimensional Laplacian operator, $f$ is the brightness distribution, and $\mu$ is the threshold.

Since the edge pattern $f_{m}$ contains noise, $f_{m}$ is represented by the following equation:

$$
\chi\left[\mathrm{f}_{\mathrm{m}}(\mathrm{t}, \mathrm{x})\right]=\chi\left[\mathrm{f}_{\mathrm{e}}(\mathrm{t}, \mathrm{x})\right]+\frac{\partial \mathrm{w}(\mathrm{t}, \mathrm{x})}{\partial \mathrm{t}}
$$

in $T \times G$,

where $\partial w(t, x) / \partial t$ denotes a white noise with respect to the time variable, $\chi[\bullet]$ is a characteristic function, and $\mathrm{f}_{\mathrm{e}}$ is an edge pattern without noise.

In this paper, if an object shape and camera parameters are known, we would like to derive the algorithm for calculating the position and orientation of that object. We obtain the position and orientation of the object when the reference pattern has been matched to the edge pattern. Thus, there exists the estimate parameter $\theta^{*}$ which satisfies

$$
\chi\left[\mathrm{f}_{\mathrm{r}}\left(\mathrm{t}, \mathrm{x} ; \theta^{*}\right)\right]=\mathrm{E}\left\{\chi\left[\mathrm{f}_{\mathrm{m}}(\mathrm{t}, \mathrm{x})\right]\right\} \quad \text { in } \mathrm{T} \times \mathrm{G},
$$

where $\mathrm{f}_{\mathrm{r}}$ is a reference pattern.

Unfortunately it is impossible to match the reference pattern to the edge pattern by making a direct, three-dimensional comparison of the points on the reference pattern with the points on the edge pattern. This is because the threedimensional points of the object cannot be defined on the edge pattern. Therefore, we tried pattern detection, using the potential field. The potential fields can be generated by diffusing the edge pattern and the reference pattern, by using the diffusion equations.

The potential field $u_{m}$ of the edge pattern is modeled by the following partial differential equation:

$$
\frac{\partial \mathrm{u}_{\mathrm{m}}(\mathrm{t}, \mathrm{x})}{\partial \mathrm{t}}-\mathrm{a} \Delta \mathrm{u}_{\mathrm{m}}(\mathrm{t}, \mathrm{x})=\chi\left[\mathrm{f}_{\mathrm{m}}(\mathrm{t}, \mathrm{x})\right]
$$

with the initial and boundary conditions

$$
\mathrm{u}_{\mathrm{m}}(0, \mathrm{x})=\mathrm{u}_{\mathrm{m} 0}(\mathrm{x})
$$

and

$$
\mathrm{u}_{\mathrm{m}}(\mathrm{t}, \mathrm{x})=0
$$

on $\mathrm{T} \times \partial \mathrm{G}$,

where a is a positive constant and $\chi\left[\mathrm{f}_{\mathrm{m}}\right]$ is a characteristic function of $\mathrm{f}_{\mathrm{m}}$.

Define

$$
\mathrm{V}=\mathrm{H}_{0}^{1}(\mathrm{G}) \subset \mathrm{H}=\mathrm{L}^{2}(\mathrm{G}) \subset \mathrm{V}^{\prime}=\mathrm{H}^{-1}(\mathrm{G}),
$$

where $\mathrm{V}^{\prime}$ is a dual space of $\mathrm{V}$.

Therefore, we can formulate the potential field (2.4) as

$$
\begin{gathered}
\left(u_{m}(t), \phi\right)+\int_{0}^{t}<A u_{m}, \phi>d s-(b w, \phi) \\
=\left(u_{m} 0, \phi\right)+\int_{0}^{t}\left(\chi\left[f_{e}\right], \phi\right) d s
\end{gathered}
$$

for $\forall \phi \in V$,

where $\mathrm{A}$ is a linear operator

$$
<A \phi, \psi>=\sum_{\mathrm{i}=1}^{2} \int_{\mathrm{G}} \mathrm{a} \frac{\partial \phi}{\partial \mathrm{x}_{\mathrm{i}}} \frac{\partial \psi}{\partial \mathrm{x}_{\mathrm{i}}} \mathrm{dx} \quad \text { for } \forall \phi, \psi \in \mathrm{V}
$$

denotes a duality product on $\mathrm{V} \times \mathrm{V}^{\prime}$, and $\mathrm{w}(\mathrm{t})$ is a Brownian motion process with values in $\mathrm{H}$ such that

$$
E\left\{|\mathrm{w}(\mathrm{t})|^{2}\right\}=\int_{0}^{\mathrm{t}} \mathrm{tr}[\mathrm{Q}(\mathrm{s})] \mathrm{ds},
$$

where $\mathrm{Q}(\mathrm{t})$ is a self-adjoint positive operator which belongs to the trace class $\mathcal{L}^{1}(\mathrm{H} ; \mathrm{H})$.

[Theorem 2.1] (Existence and Uniqueness Theorem): With the conditions

$$
\mathrm{u}_{\mathrm{m} 0} \in \mathrm{L}^{2}(\Omega, \mathrm{H})
$$

and

$$
\chi\left[\mathrm{f}_{\mathrm{e}}\right] \in \mathrm{L}^{2}(\Omega \times \Gamma ; \mathrm{H}),
$$

there exists a unique solution to (2.5) such that

$$
\mathrm{u}_{\mathrm{m}} \in \mathrm{L}^{2}(\Omega \times \mathrm{T} ; \mathrm{V}) \cap \mathrm{L}^{2}(\Omega, \mathrm{C}(\overline{\mathrm{T}} ; \mathrm{H})) .
$$

Proof: We can prove this theorem easily, by using the Picard method [4].

The potential field $u_{r}$ of the reference pattern is modeled by the following partial differential equation:

$$
\frac{\partial \mathrm{u}_{\mathrm{r}}(\mathrm{t}, \mathrm{x})}{\partial \mathrm{t}}-\mathrm{a} \Delta \mathrm{u}_{\mathrm{r}}(\mathrm{t}, \mathrm{x})=\chi\left[\mathrm{f}_{\mathrm{r}}(\mathrm{t}, \mathrm{x})\right] \quad \text { in } \mathrm{T} \times \mathrm{G},
$$

with the initial and boundary conditions

$$
\mathrm{u}_{\mathrm{r}}(0, \mathrm{x})=\mathrm{u}_{\mathrm{r} 0}(\mathrm{x})
$$

on $\mathrm{G}$ 


$$
\mathrm{u}_{\mathrm{r}}(\mathrm{t}, \mathrm{x})=0
$$

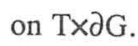

Therefore, we can formulate the potential field (2.9) as

$$
\begin{aligned}
& \left(\mathrm{u}_{\mathrm{r}}(\mathrm{t}), \phi\right)+\int_{0}^{\mathrm{t}}\left\langle\mathrm{au}_{\mathrm{r}}, \phi>d \mathrm{ds}\right. \\
& =\left(\mathrm{u}_{\mathrm{r} 0}, \phi\right)+\int_{0}^{\mathrm{t}}\left(\chi\left[\mathrm{f}_{\mathrm{r}}\right], \phi\right) \mathrm{ds}
\end{aligned}
$$

for $\forall \phi \in V$.

[Theorem 2.2] (Existence and Uniqueness Theorem): With the conditions

$$
\mathrm{u}_{\mathrm{r} 0} \in \mathrm{L}^{2}(\mathrm{G})
$$

and

$$
\chi\left[\mathrm{f}_{\mathrm{r}}\right] \in \mathrm{L}^{2}(\mathrm{~T} ; \mathrm{H})
$$

there exists a unique solution to (2.10) such that

$$
\mathrm{u}_{\mathrm{r}} \in \mathrm{L}^{2}(\mathrm{~T} ; \mathrm{V})
$$

Proof: We can prove this theorem easily, by using the Picard method.

\section{Pattern Matching Problem}

Assuming that the force $\psi(t, x)$ acts on the edge pattern near the reference pattern, and the parameter $\theta$ represents the position and orientation of a camera, $\psi(t, x)$ is represented intuitively by the following equation:

$$
\psi(t, x)=\chi\left[f_{m}(t, x)\right] \operatorname{grad} u_{r}(t, x ; \theta)
$$

in $T \times G$.

The parameter $\theta$ changes like this:

$$
\frac{\mathrm{d} \theta}{\mathrm{dt}}=\int_{\mathrm{G}} \mathrm{W}(\mathrm{x}) \psi(\mathrm{t}, \mathrm{x}) \mathrm{dx},
$$

where $\mathrm{W}(\mathrm{x})$ is a $6 \times 2$ vector field matrix specifying the direction and speed of the parameter update.

Consider the cost function

$$
\mathrm{J}(\theta(\mathrm{t}))=E\left\{\left(\chi\left[\mathrm{f}_{\mathrm{m}}\right], \mathrm{u}_{\mathrm{m}}-\mathrm{u}_{\mathrm{r}}(\theta)\right)\right\}
$$

The pattern matching problem is that the estimate parameter $\theta^{*}$ is attained by minimizing the cost function $J(\theta)$ such that

$$
\mathrm{J}\left(\theta^{*}\right)=\inf _{\theta \in \mathcal{U}_{\mathrm{ad}}} \mathrm{J}(\theta),
$$

where $u_{\mathrm{ad}}$ is an admissible class and is represented by the following equation:

$$
\mathcal{U}_{\mathrm{ad}}=\left\{\theta \in \mathrm{S}_{\mathrm{c}}: 0 \leq \theta_{\mathrm{i}}<2 \pi\left(\theta_{\mathrm{i}}=4,5,6\right)\right\}
$$

where $S_{c}$ is a set of positions and orientations of the camera when the object image is in the picture.

From the maximal principle for the diffusion equation, it is easy to verify that the cost function $J(\theta)$ is nonnegative definite and takes zero if and only if $\theta=\theta^{*}$ [2].

[Theorem 3.1] (Convergence of Parameter $\theta$ ) : if the initial value of the parameter $\theta$ exists in the image domain in which the potential field of the reference pattern is convex, and if the estimate parameter $\theta^{*}$ exists, and if the parameter update $\theta$ is given by the equation

$$
\frac{\mathrm{d} \theta}{\mathrm{dt}}=\int_{\mathrm{G}} \chi\left[f_{\mathrm{m}}(\mathrm{t}, \mathrm{x})\right]\left[\frac{\partial \mathrm{x}}{\partial \theta}\right]^{\prime} \operatorname{grad} \mathrm{u}_{\mathrm{r}}(\mathrm{t}, \mathrm{x} ; \theta) \mathrm{dx}
$$

then the parameter $\theta$ converges to the estimate parameter $\theta^{*}$ asymptotically.

Proof : the derivative of the cost function $J(\theta)$ is calculated as follows:

$$
\begin{aligned}
\frac{\mathrm{dJ}(\theta)}{\mathrm{dt}} & =E\left\{\frac{\mathrm{d}}{\mathrm{dt}} \int_{\mathrm{G}} \chi\left[\mathrm{f}_{\mathrm{m}}(\mathrm{t}, \mathrm{x})\right]\left(\mathrm{u}_{\mathrm{m}}(\mathrm{t}, \mathrm{x})-\mathrm{u}_{\mathrm{r}}(\mathrm{t}, \mathrm{x} ; \theta)\right) \mathrm{dx}\right\} \\
& =-E\left\{\left[\frac{\mathrm{d} \theta}{\mathrm{dt}}\right]^{\prime} \int_{\mathrm{G}} \chi\left[\mathrm{f}_{\mathrm{m}}(\mathrm{t}, \mathrm{x})\right]\left[\frac{\partial \mathrm{u}_{\mathrm{r}}(\mathrm{t}, \mathrm{x} ; \theta)}{\partial \theta}\right] \mathrm{dx}\right\} \\
& =-E\left\{\left[\frac{\mathrm{d} \theta}{\mathrm{dt}}\right] \int_{\mathrm{G}}^{\prime} \chi\left[\mathrm{f}_{\mathrm{m}}(\mathrm{t}, \mathrm{x})\right]\left[\frac{\partial \mathrm{x}}{\partial \theta}\right]^{\prime} \operatorname{grad} \mathrm{u}_{\mathrm{r}}(\mathrm{t}, \mathrm{x} ; \theta) \mathrm{dx}\right\} .
\end{aligned}
$$

From (3.6), the derivative of the cost function is zero or negative, as shown in the following equation:

$$
\frac{\mathrm{dJ}(\theta)}{\mathrm{dt}}=-E\left\{\left(\int_{\mathrm{G}} \chi\left[\mathrm{f}_{\mathrm{m}}(\mathrm{t}, \mathrm{x})\right]\left[\frac{\partial \mathrm{x}}{\partial \theta}\right]^{\prime} \operatorname{grad} \mathrm{u}_{\mathrm{r}}(\mathrm{t}, \mathrm{x} ; \theta) \mathrm{dx}\right)^{2}\right\} \leq 0 .
$$

From the above, if the initial value of the parameter $\theta$ exists in the image domain in which the potential field of the reference pattern is convex, and if the estimate parameter $\theta^{*}$ exists, the 
parameter $\theta$ converges to the estimate parameter $\theta^{*}$.

Next, let the parameter $\theta$ be the position and orientation of an object. Assuming a force acts on the reference pattern near the edge pattern, the force $\Psi_{\mathrm{c}}$ is represented intuitively by the following equation:

$$
\psi_{\mathrm{c}}(\mathrm{t}, \mathrm{x})=\chi\left[\mathrm{f}_{\mathrm{r}}(\mathrm{t}, \mathrm{x} ; \theta)\right] \operatorname{grad} \mathrm{u}_{\mathrm{m}}(\mathrm{t}, \mathrm{x})
$$

in $\mathrm{T} \times \mathrm{G}$.

The parameter $\theta$ changes like this:

$$
\frac{d \theta}{d t}=\int_{G} W_{c}(x) \psi_{c}(t, x) d x,
$$

where $\mathrm{W}_{\mathrm{c}}(\mathrm{x})$ is a $6 \times 2$ vector field matrix specifying the direction and speed of the parameter update. The parameter update $\theta$ is given by the following equation using the same method as above:

$$
\frac{d \theta}{d t}=\int_{G} \chi\left[f_{r}(t, x ; \theta)\right]\left[\frac{\partial x}{\partial \theta}\right]^{\prime} \operatorname{grad} u_{m}(t, x) d x
$$

An admissible class $\mathcal{U}_{\mathrm{ad}}$ is represented by

$$
U_{\mathrm{ad}}=\left\{\theta:\left(\theta_{1}, \theta_{2}, \theta_{3}\right) \in \mathrm{S}, 0 \leq \theta_{\mathrm{i}}<2 \pi\left(\theta_{\mathrm{i}}=4,5,6\right)\right\},
$$

where $S$ is a visible space from the camera.

Consequently, we attain Algorithm 3.1 of the six degrees freedom contour matching.

[Algorithm 3.1]: By iterating the following Steps 1-3, we attain the position and orientation of the object.

Step 1: Obtain the edge pattern $\mathrm{f}_{\mathrm{m}}$ from the image $\mathrm{f}$, using (2.1).

Step 2: Obtain the potential patterns $\mathrm{u}_{\mathrm{m}}$ with the edge pattern $\mathrm{f}_{\mathrm{m}}$, using (2.4).

Step 3: Obtain the parameter $\theta$, using (3.9).

\section{Approximation of Optical Flow}

In this section, the vector field matrix $\mathrm{W}_{\mathrm{c}}(\mathrm{x})$ is derived. From Section $3, W_{c}(x)$ is given by

$$
\mathrm{W}_{\mathrm{c}}(\mathrm{x})=\left[\frac{\partial \mathrm{x}}{\partial \theta}\right]
$$

where $\partial x / \partial \theta$ is a $2 \times 6$ matrix. An object coordinate system $\left(x_{0}\right.$, $\mathrm{y}_{0}, \mathrm{z}_{\mathrm{o}}$ ), a camera coordinate system $\left(\mathrm{x}_{\mathrm{c}}, \mathrm{y}_{\mathrm{c}}, \mathrm{z}_{\mathrm{c}}\right)$ and a scene coordinate system $\left(\mathrm{x}_{1}, \mathrm{x}_{2}\right)$ are defined in Fig. 4.1, where $\left(\mathrm{e}_{1}, \mathrm{e}_{2}\right)$ is a scene coordinate of camera's optical axis.

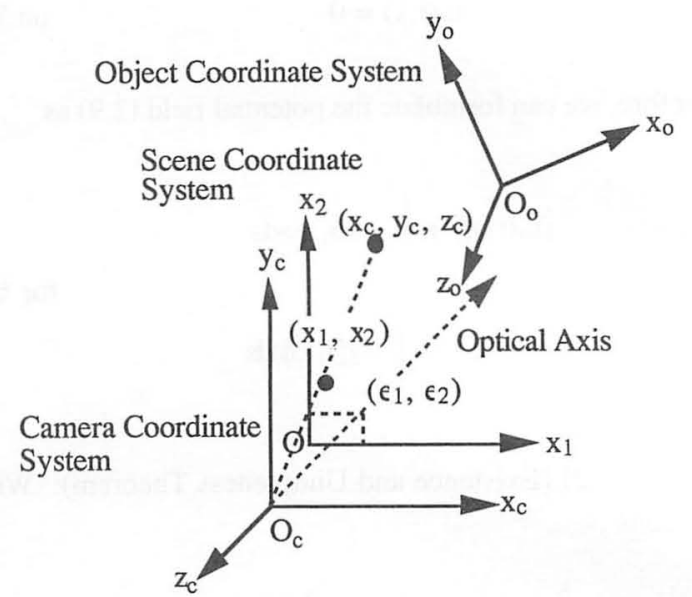

Fig. 4.1 Coordinate System of the Object, Camera and Scene

[Theorem 4.1] : The vector field matrix is represented approximately by

$$
\mathrm{W}_{\mathrm{c}}(\mathrm{x}) \cong\left[\begin{array}{cc}
\mathrm{d}_{1} \mathrm{~d}_{5} & 0 \\
0 & \mathrm{~d}_{2} \mathrm{~d}_{5} \\
-\mathrm{d}_{3} \mathrm{~d}_{5}\left(\mathrm{x}_{1}-\mathrm{e}_{1}\right) & -\mathrm{d}_{4} \mathrm{~d}_{5}\left(\mathrm{x}_{2}-\mathrm{e}_{2}\right) \\
\mathrm{d}_{3} \mathrm{~d}_{6}\left(\mathrm{x}_{1}-\mathrm{e}_{1}\right)\left(\mathrm{x}_{2}-\mathrm{x}_{\mathrm{g}}\right) & \mathrm{d}_{4} \mathrm{~d}_{6}\left(\mathrm{x}_{2}-\mathrm{e}_{2}\right)\left(\mathrm{x}_{2}-\mathrm{x}_{\mathrm{g} 2}\right) \\
-\mathrm{d}_{3} \mathrm{~d}_{6}\left(\mathrm{x}_{1}-\mathrm{e}_{1}\right)\left(\mathrm{x}_{1}-\mathrm{x}_{\mathrm{g} 1}\right) & -\mathrm{d}_{4} \mathrm{~d}_{6}\left(\mathrm{x}_{2}-\mathrm{e}_{2}\right)\left(\mathrm{x}_{1}-\mathrm{x}_{\mathrm{g} 1}\right) \\
\mathrm{d}_{1} \mathrm{~d}_{6}\left(\mathrm{x}_{2}-\mathrm{x}_{\mathrm{g} 2}\right) & -\mathrm{d}_{2} \mathrm{~d}_{6}\left(\mathrm{x}_{1}-\mathrm{x}_{\mathrm{g} 1}\right)
\end{array}\right]
$$

where $d_{i}(i=1, \ldots, 6)$ are positive constants and $\left(x_{g 1}, x_{g 2}\right)$ is a scene coordinate of an object's center of gravity

Proof: The relation between the camera coordinate system and the scene coordinate system can be shown as follows:

$$
\begin{aligned}
& \mathrm{x}_{1}=-\mathrm{c}_{1} \frac{\mathrm{x}_{\mathrm{c}}}{\mathrm{z}_{\mathrm{c}}}+\mathrm{e}_{1} \\
& \mathrm{x}_{2}=-\mathrm{c}_{2} \frac{\mathrm{y}_{\mathrm{c}}}{\mathrm{z}_{\mathrm{c}}}+\mathrm{e}_{2},
\end{aligned}
$$

where $c_{1}$ and $c_{2}$ are magnifications in the $x_{1}$ and $x_{2}$ directions respectively.

The scene coordinate $\left(\mathrm{x}_{1}, \mathrm{x}_{2}\right)$ is represented by the position and orientation $\theta$ of the object and the object coordinate $\left(\mathrm{x}_{0}, \mathrm{y}_{\mathrm{o}}, \mathrm{z}_{\mathrm{o}}\right)$. 
$(4.4)$

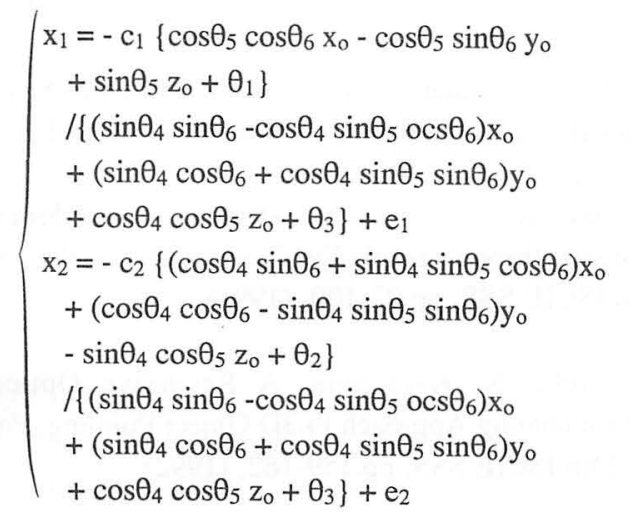

From (4.4), $\partial \mathrm{x} / \partial \theta$ is calculated:

$$
\begin{gathered}
\frac{\partial x_{1}}{\partial \theta_{1}}=-c_{1} \frac{1}{z_{0}+\theta_{3}} \\
\frac{\partial x_{2}}{\partial \theta_{1}}=0 \\
\frac{\partial x_{1}}{\partial \theta_{2}}=0 \\
\frac{\partial x_{2}}{\partial \theta_{2}}=-c_{2} \frac{1}{z_{0}+\theta_{3}} \\
\frac{\partial x_{1}}{\partial \theta_{3}}=-c_{1} \frac{x_{0}+\theta_{1}}{\left(z_{0}+\theta_{3}\right)^{2}} \\
\frac{\partial x_{2}}{\partial \theta_{3}}=-c_{2} \frac{y_{0}+\theta_{2}}{\left(z_{0}+\theta_{3}\right)^{2}} \\
\frac{\partial x_{1}}{\partial \theta_{4}}=c_{1} \frac{\left(x_{0}+\theta_{2}\right) y_{0}}{\left(z_{0}+\theta_{3}\right)^{2}} \\
\frac{\partial x_{1}}{\partial \theta_{5}}=-c_{1} \frac{z_{0}\left(z_{0}+\theta_{3}\right)+y_{0}\left(y_{0}+\theta_{2}\right)}{\left(z_{0}+\theta_{3}\right)^{2}} \\
\frac{\left.\partial x_{2}\right)+x_{0}\left(x_{0}+\theta_{1}\right)}{\left(z_{0}+\theta_{3}\right)^{2}}=-c_{2} \frac{x_{0}\left(y_{0}+\theta_{2}\right)}{\left(z_{0}+\theta_{3}\right)^{2}} \\
\frac{\partial \theta_{5}}{\partial \theta_{6}}=-c_{1} \frac{y_{0}}{z_{0}+\theta_{3}} \\
z_{0}+\theta_{3}
\end{gathered}
$$

where $\theta_{4}, \theta_{5}$ and $\theta_{6}$ are zero. Here, from coordinate transformation, we get

$$
\begin{array}{r}
\mathrm{x}_{1}-\mathrm{e}_{1} \propto \frac{\mathrm{x}_{\mathrm{o}}+\theta_{1}}{\mathrm{z}_{\mathrm{o}}+\theta_{3}} \\
\mathrm{x}_{2}-\mathrm{e}_{2} \propto \frac{\mathrm{y}_{\mathrm{o}}+\theta_{2}}{\mathrm{z}_{\mathrm{o}}+\theta_{3}} \\
\mathrm{x}_{1}-\mathrm{x}_{\mathrm{g} 1} \propto \frac{\mathrm{x}_{0}}{\mathrm{z}_{\mathrm{o}}+\theta_{3}}
\end{array}
$$

$$
\mathrm{x}_{2}-\mathrm{x}_{\mathrm{g} 2} \propto \frac{\mathrm{y}_{\mathrm{o}}}{\mathrm{z}_{0}+\theta_{3}} .
$$

From the above equations and by approximating $\left(\mathrm{z}_{\mathrm{o}}+\theta_{3}\right)$ and $\mathrm{z}_{\mathrm{d}}\left(\mathrm{z}_{\mathrm{o}}+\theta_{3}\right)$, we are able to easily arrive at (4.2).

From the vector field matrix $\mathrm{W}_{\mathrm{c}}(\mathrm{x})$, the optical flow can be represented by Fig. 4.2.

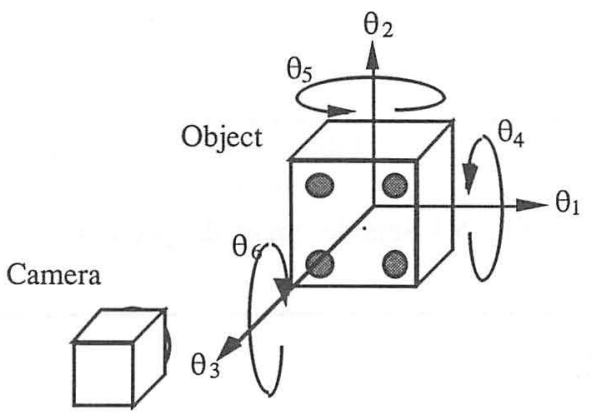

Translation of Object

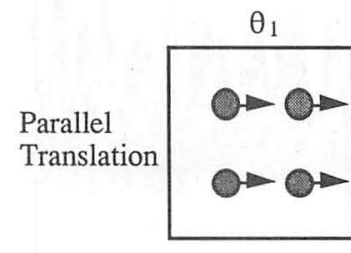

$\theta_{4}$

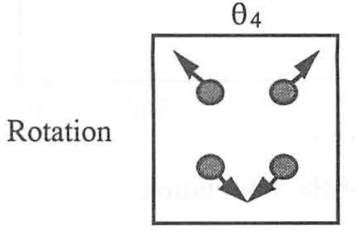

Fig. 4.2 Optical Flow

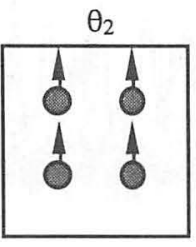

$\theta_{5}$

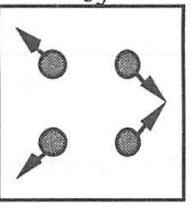

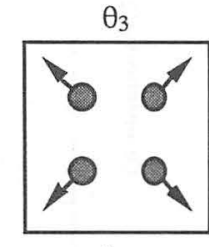

$\theta_{6}$

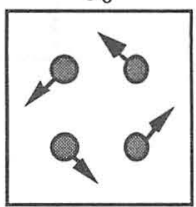

\section{Experiments}

Experiments are performed by using the finite difference method with time and image spatial variables.

In an example, the time-partition and the image spatialpartition were set as $\Delta t=1$ and $\Delta x=1$, the image region size is $512 \times 512$ and the values of parameters (3.4) and (3.7) were set as

$$
\left\{\begin{array}{l}
\mathrm{a}=0.1, \quad \chi\left[\mathrm{f}_{\mathrm{m}}\right]=0.09 \mid \operatorname{div} \mathrm{fl}, \\
\mathrm{d}_{1}=1, \mathrm{~d}_{2}=1, \mathrm{~d}_{3}=10^{-2}, \mathrm{~d}_{4}=10^{-2}, \\
\mathrm{~d}_{5}=10^{-3}, \mathrm{~d}_{6}=10^{-5}
\end{array}\right.
$$

The experiments were performed, using Algorithm 4.1. A sample run of the position and orientation of the object is plotted in Fig. 5.1 and Fig. 5.2. 


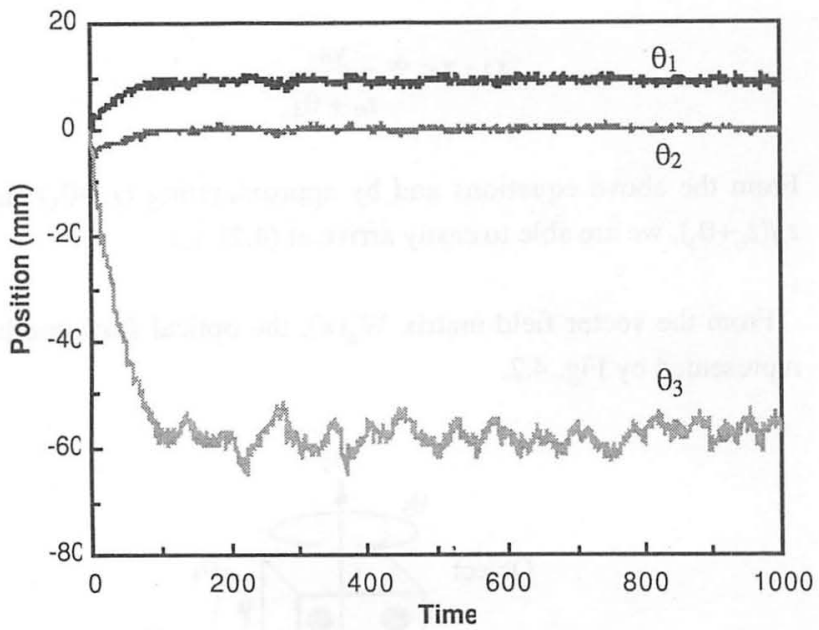

Fig. 5.1 Sample run of the position

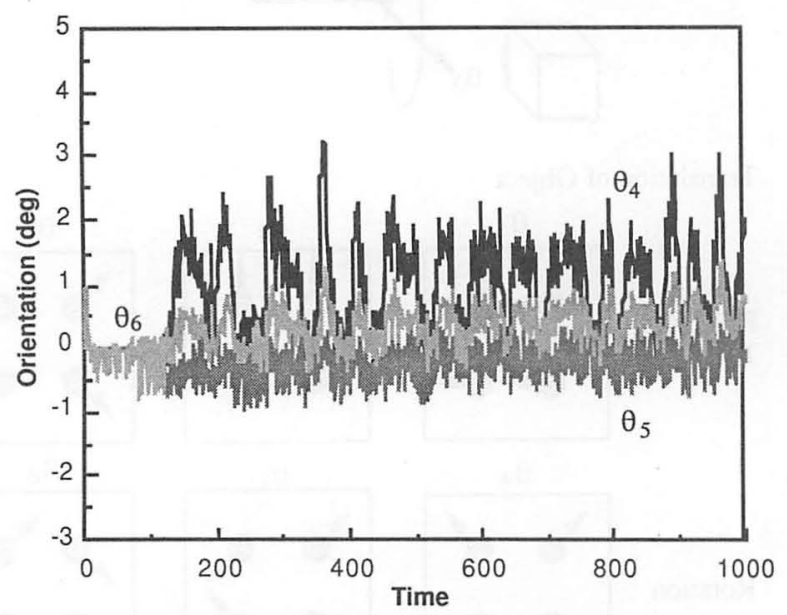

Fig. 5.2 Sample run of the orientation

\section{Conclusions}

In this paper, the pattern detection problem has been solved within the framework of pattern matching using the pattern field. Advantages of pattern detection derived here are:

(a) Pattern detection which has mathematical security can be easily implemented.

(b) We have experimentally verified advantage (a).

\section{References}

[1] D. Marr and E. Hildreth: Theory of Edge Detection, Proc. R. Soc. London B-207, pp.187-217, (1980)

[2] K. Kamejima, Y. Ogawa and Y. Nakano: A Fast Algorithm for Approximating 2D Diffusion Equation with Application to Pattern Detection in Random Image Fields, Proc. IMACS/IFAC int. SYMP. on Modeling and Simulation of Distributed Parameter Systems, (1987)

[3] T. Hamada, K. Kamejima and I. Takeuchi: Dynamic Work Space Model Matching for Interactive Robot Operation, Proc. IEEE Int. Workshop on Industrial Application of
Machine Intelligence and Vision, pp.82-87, (1989)

[4] E. Pardoux: Equations aux D riv es Partielles Stochastique Non Lin ares Monotones, Th se l'Universit de Paris, (1975)

[5] T. Aoki, K. Kamejima: Six Degrees of Freedom Contour Matching through Single Eye Noisy Imagery, Preprint of the 23th ISCIE SSS, pp.97-100, (1991)

[6] T. Aoki, K. Kamejima: A Recursive Optical Flow Approximating Approach to 3D Object Pointing, Preprint of the 24th ISCIE SSS, pp.159-162, (1992) 